\title{
WiFi Ad-Hoc Mesh Network and MAC Protocol Solution for UWB Indoor Localization Systems
}

\author{
Matteo Ridolfi, Samuel Van de Velde, Heidi Steendam, Eli De Poorter \\ Ghent University - iMinds \\ 9052 Ghent, Belgium \\ matteo.ridolfi@intec.ugent.be
}

\begin{abstract}
Ultra-wideband (UWB) is an emerging technology that is well known for its capability to provide centimeter positioning precision which is essential for applications that require very high accuracy. However, although several accuracy improving algorithms have been proposed in scientific literature, solutions to improve the scalability (in terms of coverage and dense user deployments) have not been explored yet or are still under examination. To this end, this paper presents a WiFiUWB solution in which the UWB indoor localization system is deployed on top of a WiFi ad-hoc mesh network. The combination of a high-throughput WiFi mesh backbone in combination with a high-accuracy UWB radios allows deployment in areas without existing (wired) backbones. Moreover, the possibility for the clients to move around the covered area maintaining fully connectivity (roaming feature) is implemented. Finally, to improve scalability, a Time Division Multiple Access (TDMA) MAC protocol that combines WiFi and UWB technologies is proposed, thereby improving the scalability of the network towards multiple mobile users. As such, the solution combines the strengths of the WiFi technology (high throughput and efficient multi-hop meshing with roaming) with the main strengths of UWB (high accuracy localization). Overall the system supports 100 simultaneous clients while supporting multi-hop roaming with $150 \mathrm{~ms}$ delays.

Index Terms-UWB, WiFi, Indoor Localization System,
\end{abstract} TDMA, Roaming, Arduino, Pozyx, ad-hoc Network, Mesh Network

\section{INTRODUCTION}

C Ontrary to widely spread outdoor positioning techniques like GPS, indoor localization systems have to face several difficulties, such as coping with errors due to multi-path and Non-Line-of-Sight (NLOS) conditions, obstacles causing signal scattering and mobile entities that change the propagation channel [1]. Thus, parameters such as accuracy, robustness, coverage and scalability have to be taken into account when designing localization systems. The Radio Frequency (RF) based indoor localization solutions seem to be the most promising due to their ease of installation. RF based solutions typically consist of two phases: (i) ranging and (ii) location estimation. The former is responsible for estimating the distance from the node to a well-known anchor point.

The distance from the anchor can be computed with measurements of different nature. Among the others the most common are based on: the received power level (RSSI - Received Signal Strength Indicator) and the travel time of a radio frequency wave from one transmitter to one or more receivers (ToA -
Time of Arrival and TDoA - Time Difference of Arrival). During the location estimation phase, the estimated distances are combined to pinpoint the most-likely location of the node. Again, different approaches such as fingerprinting, geometric techniques and statistical methods are possible. Alternatively, positioning estimation can be based on the angle of an incident RF waves by means of special antennas (AoA - Angle of Arrival).

These techniques can be applied to a wide range of wireless technologies:

- Bluetooth Low Energy (BLE) and IEEE 802.11 WiFi are readily available in all smartphones but suffer from low accuracy due to fluctuating signal strengths. Errors are typically in the order of several meters.

- Ultra Wide Band (UWB) offers a centimeter-level accuracy in Line-Of-Sight (LOS) conditions. In NLOS the most performant systems have a maximum error of 30 $\mathrm{cm}$ or less. However, to the best of our knowledge, there are currently no low-cost UWB systems for indoor positioning on the market that cover large multi-hop areas with dense numbers of users.

The main focus of this document is to improve the coverage and scalability of indoor localization systems using UWB technologies. The WiFi creates an ad-hoc mesh network among the fixed anchor nodes, creating a suitable infrastructure to host the UWB localization network. The ad-hoc approach is a flexible solution that facilitates the deployment of the system where there is no predefined infrastructure. In addition, WiFi is much better suited for high-throughput connections than IEEE 802.15.4a UWB, allowing each mobile node to connect to the WiFi backbone for receiving content and transmitting location estimates to a central server. A TDMA protocol is designed wherein each mobile node is assigned an UWB slot for ranging, thereby avoiding collisions and improving scalability. The cooperation between these two technologies results in a new WiFi-UWB MAC protocol. The remainder of the paper is organized as follows. Section II describes related work. Afterwards, in Section III, the system architecture is introduced. The following two sections are reserved to describe and analyze the performance of the MAC protocol and the network layer respectively. The integrated solution along with test results is illustrated in the section VI. Finally, 
the conclusion is described in section VII, followed by the future work.

\section{RELATED WORK}

\section{A. Indoor Localization Systems}

In literature there is a significant number of publications on indoor localization technologies proving that different localization approaches are possible. For example, in [2] a WiFi-based approach is described. Simulations show that this positioning system can achieve around $1 \mathrm{~m}$ accuracy without any hardware change in commercial $\mathrm{WiFi}$ products but relying on wide signal bandwidth and large numbers of antennas by utilizing the transmission of multiple predefined messages . Even if 1 $\mathrm{m}$ accuracy might sound quite accurate, in many scenarios this is just not enough. Higher accuracies can be obtained using techniques such as UWB. A pure UWB solution for indoor robot navigation is designed in [3]. The system tracks one robot in a 3 cells area. They obtained a maximum error of $25 \mathrm{~cm}$ using a wired backbone to connect the infrastructure anchor points. To further improve the accuracy, sensor fusion with other sensor technologies is a viable solution as shown in [4]. In this paper, the authors presented an architecture to collect data from different types of sensors in order to improve the accuracy of the UWB localization.

Although many paper focus on increasing the accuracy of UWB solutions, most of these papers consider a simple set-up with UWB anchor nodes covering a single collision domain wherein a single mobile device is tracked. For commercial deployment, two further improvements are required: (i) improving the scalability (i.e. the coverage and supported node density) of an UWB positioning system and (ii) providing a more flexible infrastructure solution. With this in mind, the main contribution of this paper is the combination of $\mathrm{WiFi}$ and UWB technologies to overcome the insufficient WiFi accuracy and the challenging UWB scalability in a scenario without a predefined infrastructure.

\section{B. UWB MAC protocols}

Because the low transmission power used in UWB is below the noise floor, it is often difficult to detect the presence of a carrier signal using a conventional carrier sensing schemes [5], thereby limiting the usefulness of CSMA/CA. To bypass this problem a standard has been defined: IEEE 802.15.4-2011 [6]. This version includes the work of the Task Group 802.15.4a which added UWB and CSS PHY's to the IEEE 802.15.4. Since carrier sensing cannot be used, the standard defines ALOHA protocol as suggested approach. ALOHA is a random access protocol and as such is not suitable when many users are in the network. TDMA techniques can be used to improve the users density. Such TDMA-based protocols allocate one or more exclusive free collision time-slots to each node for communication, depending on the scheduling algorithm.

In the scientific community, several improvements have been suggested to enhance the performance of UWB MAC protocols. In [7], a MAC protocol called UCAN is developed. It uses localization properties to manage the access and the routing in the network. MAC and routing protocols are described but the piconet approach works only for WPAN's and therefore it is not suitable for large networks. A proactive and adaptive MAC protocol (U-MAC) is proposed in [8]. The authors considered three different topologies with 25 nodes each to investigate the symmetric UWB traffic case. The protocol framework provides measures of positional reliability, but their simulations only considered stationary nodes and single hop scenario. In UMAC the power is varied depending on the interference signal to noise ratio while in [9], a distributed protocol for very low power UWB devices is described. They designed a MAC protocol based on dynamic channel coding. It is claimed that the main advantage of this approach is that the MAC adapts to the varying channel, therefore supporting medium mobility levels and ad-hoc mode. To isolate the effect of mobility they have not use multi-hop routing. A different approach is presented in [10], in which a multi-band UWB MAC ad-hoc network is created. They distinguish between data bands and request bands, each of $500 \mathrm{MHz}$ bandwidth. Routing, flow control or multiple service classes are not investigated and the synchronization is assumed to be present in every packet which results in high overhead.

Due to the combination of WiFi and UWB, the approach presented in this work removes the "contention period" that typically precedes the actual data slots in any TDMA classic approach. Except for a beacon interval used for synchronization purposes, the rest of the superframe is entirely dedicated to the data transmission and this will improve the users density because the users do not have to compete anymore.

\section{SYSTEM ARCHITECTURE}

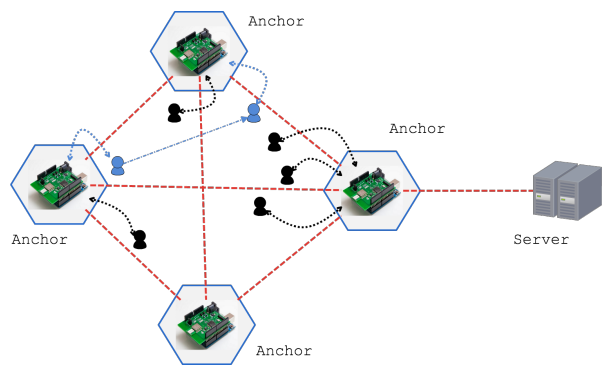

Fig. 1: Overall system overview - WiFi

This section describes the overall architecture of the localization solution (Figure 1).

Except for the server, every node in the network consists of two boards (Pozyx [11] and Arduino Yún [12]) with two different technologies enabled, UWB and WiFi respectively. As such, each node has two hardware elements (Figure 2), where the Pozyx board is plugged on the Arduino as an external shield. In this way the node works both with the UWB and the WiFi antenna. More precisely Pozyx uses the Decawave DWM1000 module [13] for UWB communication, while Arduino Yún is based on the classic microcontroller ATmega32u4 [14] and the Atheros AR9331 [15] processor with a built-in IEEE $802.11 \mathrm{~b} / \mathrm{g} / \mathrm{n}$ WiFi capability. 


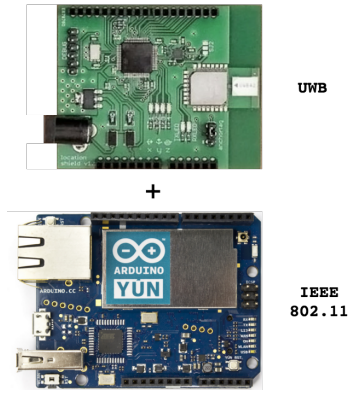

Fig. 2: Pozyx and Arduino Yún boards

Each of these nodes can take any of the following roles, as shown in Figure 1: the anchor nodes, the server (together they are part of the infrastructure) and the client nodes (tags). As depicted in Figure 1, the IEEE 802.11 WiFi network is divided in an infrastructure network and a client network. The former connects the anchors in a mesh network and then to a centralized server. The second WiFi network is the one that allows the users to be connected to the system. For this reason, each anchor point also acts as an access point (AP) towards this client network. The WiFi network is used to orchestrate the assignation of UWB slots for each client tag, this way avoiding collisions between UWB messages. The communication flow is started by the client tag. It sends a message requesting for the permission to transmit its UWB packets to the anchor nodes to calculate its position. The message is collected by the WiFi component of the infrastructure node, and forwarded over the WiFi mesh network to the central server that is in charge of assigning the medium resources. After checking which slots are available, the response with assigned slots is sent over WiFi back to the client. When the slot is received by the Linux microprocessor of the Arduino, this is communicated to the ATmega microcontroller, which makes the results available for the UWB shield. The client then synchronizes with the anchor nodes using the UWB radio, and uses the assigned slots to perform the ranging and localization measurements exchanging messages with the 4 anchor points (3D positioning). The next section describes in more detail the MAC protocol that exploits the ad-hoc mesh network to assign the resources to the UWB nodes in the system.

\section{MAC PROTOCOL}

In a standard TDMA-based solution, even if the data transmission is ensured to be collision free, a contention period to gain the right to transmit is still necessary. The solution presented in this paper removes completely the contention period because the slot assignation is managed by the WiFi network. In Figure 3(b) it is depicted the new TDMA approach. Contrary to Figure 3(a), the UWB superframe consists only of Contention Free Period (CFP) slots, removing the Contention Access Period (CAP) which follows the Beacons (B) in a classic TDMA protocol.

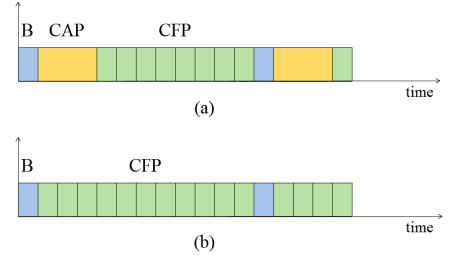

Fig. 3: UWB Time allocation

\section{A. Design}

The TDMA MAC protocol is made of two phases. The first phase is the standard IEEE 802.11 WiFi association and authentication procedure. Once this part is correctly completed, it is the client that has to start the reservation phase, meaning that it sends the reservation message that will reach the server through the anchor point. When a node sends a request to the anchor point, the server will assign the slots to the new node if there are any available.

The UWB communication is divided in superframes of 10 seconds and each superframe is in turn divided in $100 \mathrm{~ms}$ slots. This choice has been made to have long range communication using a data rate of $110 \mathrm{kpbs}$. A low value for the data rate allows to have a larger localization range but the transmission takes more time. The transmission time is also influenced by the preamble length, currently fixed at 1024 symbols. The Pozyx configuration used takes around $25 \mathrm{~ms}$ to range with one anchor.

During these $100 \mathrm{~ms}$, the tag exchanges UWB localization messages with the 4 Pozyx anchors in a Two-Way ranging scheme, which results in maximum 100 supported tags per superframe. While the $\mathrm{WiFi}$ radios of the Arduino Yúns are communicating with each other in order to obtain the right to transmit in the next superframes. After requesting UWB slots (using the WiFi network) in the n-th superframe, during the next $(n+1)$-th superframe, the client Pozyx will communicate with the anchors. This will happen only during the assigned time slots. At this point, assumed that all the nodes in the network are synchronized, at the beginning of the next superframe, the Pozyx tag will know exactly which slots are reserved for the ranging protocol and it will transmit only at those specific moments.

\section{B. Packet Format}

The WiFi packets exchanged in the MAC protocol, are built using the Scapy [16] tool. Scapy is a powerful framework to design new protocol layers and it is very flexible for adding or removing packet fields. The Slot Reservation packet (Figure 4(a)) is composed as follows:

- Type of Reservation (1 bit):

Client tags can request a One Time request (0) or a Periodic Slot (1).

- Frequency of update / Timing (32 bits):

If the request is periodic, then this field represents the ranging frequency that is requested. Otherwise it represents the time after which a slot is requested. For instance, 

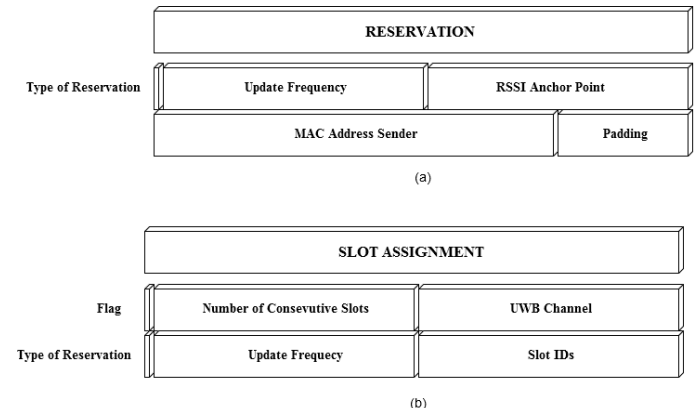

Fig. 4: WiFi packets formats for (a) requesting ranging slots and (b) slot assignment messages.

the combination $(0,0)$ for fields 0 and 1 respectively represents a user that is asking for the first available slot in the following superframe.

- RSSI of the Anchor Point (32 bits):

The Received Signal Strength Indicator of the WiFi anchor point to which the tag is currently connected. This information is taken into account for the roaming procedure.

- MAC Address Sender (48 bits):

The MAC address of the tag.

In response to the above request, the server sends the WiFi Slot Assignment packet which is depicted in Figure 4(b):

- Flag (1 bit):

To indicate whether the tag will be receiving or transmitting during the next superframe. Data aggregation between tags is also possible.

- Number of consecutive slots (32 bits):

Number of consecutive slots that are assigned. This is necessary to support ranging with a dynamic number of anchor nodes.

- UWB channel (Transmission frequency) (32 bits):

UWB radio frequency (channel) that should be used for the ranging messages; 6 channels are available.

- Type of Reservation (1 bit): One time assignation (0) or Period Assignation (1).

- Update Frequency / Timing (32 bits)

- SLOT ID (32 bits):

The unique slot ID for the next superframe (to ensure packet delays do not result in overlapping assignments).

These two packets are encapsulated into an IP packet and then sent over the TCP socket that the client establishes with the server.

\section{NETWORKING}

The infrastructure network is divided into two parts.

- The WiFi ad-hoc mesh network of the anchor points.

- The WiFi access network to connect the non-mesh client tags.

\section{A. Ad-Hoc Mesh Network}

The anchor points form a wireless ad-hoc mesh network where all nodes cooperate in the distribution of data. To create the mesh network, the protocol called Better Approach To Mobile Ad hoc Networking (B.A.T.M.A.N.) has been used, a routing protocol for multi-hop ad-hoc mesh networks [17]. The protocol version used in this project is B.A.T.M.A.N. Advanced (batman-adv). It is an implementation of the B.A.T.M.A.N. routing protocol in the form of a Linux kernel module operating at layer 2. Essentially, in a mesh network adopting this protocol, every mesh node has its own list containing all the other nodes participating to the mesh. Furthermore for each of the entry of this table, it indicates the best next-hop to reach them. There is no need to compute the entire route toward the other nodes. The so called transition table is populated thanks to special packets broadcasted from every node: the Originator Message (OGM).

\section{B. Roaming Mechanism}

The internal transition table update frequency is an interesting parameter to study the performance of the system because it affects mostly how fast it reacts to certain events. Decreasing the updating interval will generate more traffic in the network, thus the overhead will increase. But there is also a significant benefit coming from a high frequency value, which is related to the mobility of the users. Batman-adv has been chosen because it allows roaming of non-mesh clients. This is a crucial feature because it enables the users to move around the covered area without losing WiFi connectivity. The roaming mechanism uses a specific packet type called "Roaming Advertisement" which is sent whenever a node detects a roaming event. When an anchor detects a new client, it will verify first if it can communicate with it. If it can, it will enter in the roaming phase, this indicates that the user is known to the network and it is not a new client. Subsequent there is need to communicate with the old mesh node (the old anchor point) to report that the client node has moved. This is what the "Roaming Advertisement" packet is. This packet is sent in unicast to the old mesh node, which, when receiving it, will interpret this packet as a removal event in its transition table and will adopt this change in the next OGM.

\section{Non-mesh network}

The system also allows connectivity with clients who are not using the batman-adv protocol and who thus cannot join the mesh network directly. These clients are also known as mesh agnostic clients. The access to the mesh network is provided with a virtual WiFi network interface bridged to the batmanadv mesh interface. Every packet in or out the non-mesh interface, is forwarded to the batman-adv interface and then routed according to the routing protocol, by the infrastructure nodes.

\section{Round Trip Time}

The round trip time (RTT) represents the time that a packet from a client tag requires to reach the central server plus the time it takes for an acknowledgment of that packet to be received by the client tag. The overall RTT depends on the number of intermediate devices through which data must pass 
between source and destination, i.e. the hop count. The best possible scenario is 1-hop case, while the worst possible case is when the packet from the client has to go through all the anchors to reach the server. In the experiments performed, the client tag was at most 4 hops removed from the client.

The measurements have been carried out with the ping command which is very suitable for the RTT measurements since it provides automatically the minimum, average and maximum RTT values. The anchors were placed in a classic office environment, spread among several rooms. The average distance between the anchors was $10 \mathrm{~m}$ in a covered area of $130 \mathrm{~m}^{2}$. This configuration allows to avoid direct connectivity between the client and the destination node (server). Moreover, to be able to maintain the same configuration, the transmit power of the anchors was set to the minimum level $(0 \mathrm{dBm})$. In this way it was also ensured that the closest anchor would always be the best next hop. The transmit power of the the client was also set to $0 \mathrm{dBm}$. The test has been repeated 3 times with different payload sizes.

As expected, the RTT increases with the number of hops. The average RTT in $\mathrm{ms}$ is reported in Table I. In realistic scenarios, the RTT is typically lower than $250 \mathrm{~ms}$, even for 5-hop networks.

\begin{tabular}{|c|c|c|c|}
\hline N Hops & 20 Bytes & 64 Bytes & 132 Bytes \\
\hline 1 & 104,8 & 109,9 & 111,4 \\
\hline 2 & 109,8 & 127,1 & 128,3 \\
\hline 3 & 110,3 & 113,9 & 122,5 \\
\hline 4 & 114,9 & 115,6 & 116,6 \\
\hline
\end{tabular}

TABLE I: Average RTT in ms for different numbers of hops between the client node and the central server.

\section{E. TCP Throughput}

In literature there are many examples that show how the pernode throughput decreases as the number of hops increases. In [18] for example, it is shown that in a wireless ad-hoc network of $n$ nodes the throughput attainable for each node in a random scenario is $\mathcal{O}(1 / \sqrt{n \log n})$, and it scales as $\mathcal{O}(1 / \sqrt{n})$ when the circumstances are optimal i.e. in arbitrary ad hoc network. The results suggest that the capacity of each node diminishes as $n$ goes large. These results are in line with what proved in [19]. To evaluate the mesh throughput of the system a throughput test has been performed using the open source iPerf tool [20]. It allows to send TCP streams with a configurable window size to evaluate the maximum reliable throughput. The measured throughput is shown in Table II and varies between $7.99 \mathrm{Mbit} / \mathrm{sec}$ and $1.7 \mathrm{Mbit} / \mathrm{sec}$ depending on the number of hops. These results also confirm the expectations that every time one hop is added, the bandwidth is almost halved.

\section{F. Roaming Delay}

Finally, the clients can roam between different anchor nodes. Whenever a device moves to a different coverage area, it has to request new UWB slots from the central server. To allow continuous location estimates, it is important to identify the typical roaming times (i.e. the time before a new slot

\begin{tabular}{|c|c|c|c|}
\hline N Hops & $\begin{array}{c}\text { Interval } \\
\text { sec }\end{array}$ & $\begin{array}{c}\text { Data Transferred } \\
\text { Mbytes }\end{array}$ & $\begin{array}{c}\text { Bandwidth } \\
\text { Mbits/sec }\end{array}$ \\
\hline 1 & 120,3 & 115 & 7,99 \\
\hline 2 & 120,8 & 55,8 & 3,87 \\
\hline 3 & 121,3 & 34,1 & 2,36 \\
\hline 4 & 121,4 & 24,8 & 1,71 \\
\hline
\end{tabular}

TABLE II: Maximum Throughput

request can be send and as such during which no location updates can be provided). The evaluated scenario involves three devices: a client and two anchor points (A1 and A2). The client is initially associated to A1 and is moving towards A2. When it decides to switch from the old anchor to the new one, it starts a new association procedure that begins with the authentication request sent from the client to A2. When the authentication phase is completed, the reassociation starts and it follows the same pattern of the standard procedure: the client sends the request to the new anchor which replies with the association request. At this point, the batman-adv protocol sends a BATADV_UNICSAT_TVLV message which is sent from $\mathrm{A} 2$ to $\mathrm{A} 1$ to inform the last one that the client, marked as roam client, has moved to $\mathrm{A} 2$. The roaming delay is computed as the interval from the association request to the new AP, until the batman-adv unicast message reaches the old anchor. The average roaming delay that was observed is $17.157 \mathrm{~ms}$, which is an acceptable duration to be without location updates for most localization applications. The overall duration that a client tag does not provide location updates is equal to the roaming time + the round trip time required for requesting new slots. Since the round trip time (see Section V.D) is always less than $130 \mathrm{~ms}$, the overall interruption for providing position updates is at most $150 \mathrm{~ms}$, even for devices that are 4 hops removed from the central server.

During the roaming procedure, it is possible that WiFi packets get lost. To evaluate the packet loss the following scenario was created. The client was randomly moving around a $4.5 \times 5$ $\mathrm{m}^{2}$ room where four anchors were placed. With a 2 seconds period, the client was scanning the wireless spectrum to look for the highest AP and while doing this, it was pinging A1, which is the initial anchor to which it was connected. Randomly roaming for the entire duration of the ping test (40 ping messages were sent), each time it followed a random pattern; at times from $\mathrm{A} 1$ to $\mathrm{A} 2$, then to $\mathrm{A} 4$; from $\mathrm{A} 1$ to $\mathrm{A} 3$ and so on. In Table III, the behaviour of a roaming non-mesh

\begin{tabular}{|c|c|c|c|}
\hline $\begin{array}{c}\text { OGM Period } \\
\mathrm{ms}\end{array}$ & $\begin{array}{c}\text { Average Number lost } \\
\text { packets during roaming }\end{array}$ & $\begin{array}{c}\text { Reassociation } \\
\mathrm{ms}\end{array}$ & $\begin{array}{c}\text { Delta } \\
\mathrm{ms}\end{array}$ \\
\hline 100 & 0,5 & 29,8 & 2,7 \\
\hline 500 & 1,5 & 31,7 & 2,9 \\
\hline 1000 & 1,6 & 27,4 & 6,1 \\
\hline
\end{tabular}

TABLE III: Roaming Delays

client is summarized in terms of packet loss, roaming delay and delta, where delta is the time between the Reassociation Response and the batman-adv roaming message. The time necessary to obtain the reassociation response from the new anchor point is independent of the OGM period since it is 
consequence of the standard WiFi association procedure and it does not involve batman-adv. However, the OGM period influences the speed with which the new access point informs the old one about the occurred roaming. The faster response in the $100 \mathrm{~ms}$ case with respect of the $1 \mathrm{~s}$ configuration, produces also a smaller packet loss. This result was expected because, increasing the OGM period means essentially that the transition table at each node is updated less frequently. Therefore a client that has left one node and moved to another one, would be found with more delay.

\section{INTEGRATED SOLUTION}

The previously described MAC and network aspects were combined and a performance test was conducted for around 15 minutes in a $12 \times 4 \mathrm{~m}^{2}$ room, during which the tags were positioning with the fixed anchors set. The tags were able to obtain slots, they could transmit and do positioning with the anchors, avoiding any collision. The ranging measurements performed with a single anchor has a resolution of $4.69 \mathrm{~mm}$. The average positioning error for the two tags were compared with the position measured before the test with a laser meter (which has an accuracy of $1,5 \mathrm{~mm}$ up to $30 \mathrm{~m}$ ). Tag1 average error is $267 \mathrm{~mm}$ while Tag2 has an average error of $369 \mathrm{~mm}$. Considering the LOS conditions with people moving in the room and the obtained median values, the positioning was in line with the claimed UWB technology accuracy.

\section{CONCLUSION}

Although a significant body of scientific results describe improvements towards the accuracy of UWB localization solutions, scientific research focusing on improving the coverage and scalability of UWB localization systems is lacking. To remedy this, this paper describes an innovative localization solution that combines two different technologies, namely $\mathrm{WiFi}$ and UWB, to increase the number of potential users as well as the coverage of the supported network. The ad-hoc mesh network does not require a pre-existing infrastructure and is used to connect roaming client tags with a central server. Through the WiFi mesh network, communication with the client tags is possible. In addition, the WiFi network is used for exchanging control messages with the client tags, such as UWB slot requests and UWB slot assignments. As a result, the full capacity of the UWB radios are used for localization purposes.

It has been shown how the scalability is related to the design choices, for example superframe duration and the users' request (update frequency). Up to 100 client tags can request their own location update interval and corresponding UWB ranging slots are provided accordingly. Moreover, roaming between different anchor points is supported, and during the roaming procedure, communication and location updates are interrupted for at most $150 \mathrm{~ms}$ in a 4-hop network. Finally, the spatial accuracy of the UWB algorithm (in LOS conditions) is typically below $30 \mathrm{~cm}$.

\section{FUTURE WORK}

These results show that UWB localization can be used as a scalable localization solution, even for large areas with many nodes. Future work will focus on further improving the slot assignment algorithm, developing the roaming procedure by taking into account the current position of each client tag and increasing the scalability by reducing the superframe duration (e.g. by using smaller packet sizes for ranging) as well as to increase the robustness by using multiple simultaneous UWB radio frequencies for ranging.

\section{ACKNOWLEDGEMENTS}

The authors wish to acknowledge the iMinds LUNAR project that focuses on the design of scalable UWB localization solutions for retail applications.

\section{REFERENCES}

[1] A. Lo, A. Yarovoy, T. Bauge, M. Russell, D. Harmer and B. Kull, "An Ultra-wideband ad hoc sensor network for real time indoor localization of emergency responders", Delft University of Technology, Thales Research and Technology Limited and IMST GmbH, 2011

[2] Chouchang Yang and Huai-Rong Shao, "WiFi-Based Indoor Positioning", IEEE Communications Magazine, March 2015

[3] Sivanand Krishnan, Pankaj Sharma, Zhang Guoping and Ong Hwee Woon, "A UWB based Localization System for Indoor Robot Navigation", Institute for Infocomm Research, Singapore, 2007

[4] Riccardo Minutolo and Luca Alfredo Annoni, "Indoor Localization with multi sensor data fusion in ad hoc mobile scenarios", IEEE International Conference on Ultra-WideBand (ICUWB), 2014

[5] Manon Kok, Jeroen D. Hol and Thomas B. Schön, "Indoor Positioning Using Ultrawideband and Inertial Measurements", Vehicular Technology, IEEE Transactions on, pp. 1293-1303, 2015

[6] IEEE 802.15.4-2011 Standard, https://standards.ieee.org/findstds/ standard/802.15.4-2011.html

[7] Fabrice Legrand, Isabelle Bucaille, Serge Héthuin, Luca De Nardis, Guerino Giancola, Maria-Gabriella Di Benedetto, Ljubica Blazevic and Philippe Rouzet, "U.C.A.N.s Ultra Wide Band System: MAC and Routing protocols", European funded U.C.A.N. project, 2003

[8] Raja Jurdak, Pierre Baldi and Cristina Videira Lopes, "U-MAC: a proactive and adaptive UWB medium access control protocol", Wireless Communications and Mobile Computing Journal, 2005

[9] Jean-Yves Le Boudec, Ruben Merz, Bozidar Radunovic and Joerg Widmer, "DCC-MAC: a decentralized MAC protocol for 802.15.4a-like UWB mobile ad-hoc networks based on dynamic channel coding", Broadband Networks, 2004. BroadNets 2004. Proceedings. First International Conference on

[10] Ioannis Broustis , Srikanth Krishnamurthy, Michalis Faloutsos , Mart Molle and Jeffrey Foerster, "A Multiband MAC Protocol for Impulsebased UWB Ad Hoc Networks", Sensor and Ad Hoc Communications and Networks, IEEE SECON, 2005.

[11] Pozyx Labs, https://www.pozyx.io

[12] Arduino, https://www.arduino.cc

[13] ScenSor DWM1000 Module, http://www.decawave.com/products/ dwm1000-module

[14] ATmega microcontroller, http://www.atmel.com/Images/Atmel-7766-8bit-AVR-ATmega16U4-32U4_Datasheet.pdf

[15] Atheros System-on-a-Chip, https://www.openhacks.com/ uploadsproductos/ar9331_datasheet.pdf

[16] Scapy Framework, http://www.secdev.org/projects/scapy/doc/build_dissect.html

[17] B.A.T.M.A.N protocol, https://www.open-mesh.org/projects/open- mesh/wiki

[18] P. Gupta and P. R. Kumar, "The Capacity of Wireless Networks", IEEE Transactions on Information Theory, March 2000

[19] P. C. Ng and S. C. Liew, "Throughput Analysis of IEEE802.11 MultiHop Ad Hoc Networks", IEEE/ACM Transactions on Networking, 2007

[20] Iperf Network Analyzer Tool, https://iperf.fr 